\title{
Aushöhlung der Unschuldsvermutung durch gezielte Öffentlichkeit?
}

\begin{abstract}
Summary
"Targeted publicity" is apparently a new accompanying phenomenon of modern criminal proceedings. It is a term used to describe how the organs of criminal justice, media, or private entities purposefully turn to the media during ongoing criminal investigations in order to instrumentalize the serious criticism of the criminal proceedings provoked by this for purposes outside of the proceedings. Targeted publicity opposes the principle of the presumption of innocence and is thus unacceptable. This contribution analyzes targeted publicity using three current and prominent criminal proceedings of varying forms: the public staging of an arrest (the case of Benaissa), limitless reporting by the media (the case of Kachelmann), and the call for mob law (the case of Emden). The examples underscore the point that targeted publicity needs more attention in order to prevent the erosion of the principle of the presumption of innocence in the age of criminal trials by the media.
\end{abstract}

\section{Résumé}

La notion de "public ciblé » est un phénomène connexe aux procédures pénales modernes de plus en plus important. Elle désigne la situation dans laquelle les organes répressifs, les médias ou les personnes privées s'adressent de manière ciblée au public dans le cadre d'une enquête criminelle en cours afin d'instrumentaliser la stigmatisation de la procédure pénale à des fins étrangères à la procédure. Cette pratique entre en conflit avec la présomption d'innocence et n'est donc pas autorisée. L'article analyse différentes formes de celle-ci en s'appuyant sur trois éminentes procédures pénales actuelles : la mise en scène publique de l'arrestation (affaire Benaissa), la médiatisation démesurée (affaire Kachelmann) et l'appel au lynchage (affaire Emden). Lesdits exemples soulignent la nécessité de prêter une attention accrue au phénomène si l'on veut empêcher que la présomption d'innocence ne soit sapée, à l'ère de la médiatisation des procédures pénales.

\section{Das Problem}

Der moderne Strafprozess gerät auch und immer stärker zum Medienprozess. Zwar sind die spektakulären Fälle der Strafjustiz („,causes célèbres“) seit jeher fester Bestandteil 
der Presseberichterstattung. ${ }^{1}$ Nachdem aber selbst früher so verschlossene Behörden wie die Staatsanwaltschaft offensive Medienarbeit betreiben ${ }^{2}$, ist die Litigation-PR aller Verfahrensbeteiligten zu einem bestimmenden Kennzeichen aufsehenerregender moderner Strafprozesse geworden. ${ }^{3} \mathrm{Zu}$ beobachten ist dabei, dass die Medienöffentlichkeit sich nicht mehr nur auf das Hauptverfahren fokussiert. Sie begleitet häufig bereits den Beginn der Strafverfolgung oder einzelne Ermittlungsmaßnahmen, ja tritt mittlerweile auch weit im Vorfeld des Strafverfahrens auf. ${ }^{4}$ Vereinzelt wird die Kriminalität sogar öffentlich produziert, so wenn ein Privatfernsehsender auf die öffentliche Jagd nach mutmaßlichen Pädophilen im Internet geht (,Tatort Internet“ $).^{5}$

Auf diesem Hintergrund eines zunehmend veröffentlichten Ermittlungsverfahrens verdient ein Phänomen besonderes Interesse, das als ,gezielte Öffentlichkeit“ des Strafverfahrens beschrieben werden kann. Gemeint ist damit, dass Strafverfolgungsorga$\mathrm{ne}^{6}$, aber auch Medien und Private sich vor Eröffnung des Hauptverfahrens, insbesondere während eines laufenden Ermittlungsverfahrens gezielt an die Öffentlichkeit wenden, um die Prangerwirkung des Strafverfahrens zu verfahrensfremden Zwecken zu instrumentalisieren. Sichtbar geworden ist eine solcherart ,gezielte Öffentlichkeit“ zuletzt bei der Verhaftung von Nadja Benaissa, im Strafverfahren gegen Jörg Kachelmann und beim Aufruf zur Lynchjustiz im Emder Mordfall. Soweit die Instrumentalisierung der Prangerwirkung im Mittelpunkt steht, ist die ,gezielte Öffentlichkeit“ im hier verstandenen Sinne abzugrenzen von dem Phänomen, dass bearbeitende Staatsanwälte oder deren unmittelbare Vorgesetzte Ermittlungsergebnisse den Medien „stecken“, „um Anklage- oder Beerdigungsversuche auf den oberen Etagen zu verhindern oder zu erschweren“. Wagner nennt das „Durchstecherei“. ${ }^{\circ}$

Dass eine gezielte, die Prangerwirkung des Strafverfahrens instrumentalisierende Öffentlichkeit mit der Unschuldsvermutung in Konflikt gerät, liegt auf der Hand. Im Folgenden wird deshalb zunächst der (nicht unzweifelhafte) Gehalt der Unschuldsvermutung rekonstruiert, soweit er die Publizität vor Eröffnung des Hauptverfahrens betrifft (unten II.). Auf dieser Basis erfolgt eine kritische Analyse des Phänomens der, ,gezielten Öffentlichkeit" anhand der drei Beispielsfälle Benaissa, Kachelmann und Emden (unten III.). Sie macht deutlich, dass dem Trend zur, ,gezielten Öffentlichkeit“"Grenzen gesetzt werden müssen, soll die Unschuldsvermutung in der Mediengesellschaft nicht vollends ausgehöhlt werden.

1 Stellvertretend Eb. Schmidt, Justiz und Publistik, 1968; R. Hamm, Große Strafprozesse und die Macht der Medien, 1997; Friedrichsen/Gerhardt, in: Bockemühl (Hrsg.), Handbuch des Fachanwalts Strafrechts, 5. Aufl. 2012, 10. Teil, Kap. 2.

2 Dazu nur Raschke ZJS 2011, 38 und Neuling, Inquisition durch Information, 2005, S. 169 ff.

3 O. Hohmann NJW 2009, 881; Boehme-Neßler ZRP 2009, 228; Trüg NJW 2011, 1040; Holzinger/Wolff, Im Namen der Öffentlichkeit, 2009. Krit. Zabel GA 2011, 347. Vgl. zur Gleichzeitigkeit der gegenläufigen Entwicklung einer Verheimlichung des Strafverfahrens aber Marxen GA 2013, 99 (102 ff.).

4 So geschehen etwa im Fall von Christian Wulff. Marxen GA 2013, 102 spricht insoweit von einem öffentlichen „Vorvorvorverfahren“ und allgemein (101 f.) von einer Vorverlagerung der Öffentlichkeit.

5 Krit. Zabel GA 2011, 353 f., 359 ff.; Marxen GA 2013, 101.

6 Siehe dazu bereits krit. Boehme-Neßler ZRP 2009, 228 (229f.); Neuling (Fn. 2), S. 169 ff. (217 ff.).

7 Wagner, Strafprozessführung über Medien, 1987, S. 35. 


\section{Unschuldsvermutung und Publizität vor Eröffnung des Hauptverfahrens}

Nach dem Grundsatz der Unschuldsvermutung, wie er u.a. in Art. 6 Abs. 2 EMRK Ausdruck gefunden hat ${ }^{8}$, gilt jeder, der wegen einer Straftat angeklagt ist, bis zum gesetzlichen Nachweis seiner Schuld als unschuldig.

\section{Die Rechtsprechung des Bundesverfassungsgerichts und des Bundesgerichtshofs}

Das Bundesverfassungsgericht misst der Unschuldsvermutung als besonderer Ausprägung des Rechtsstaatsprinzips Verfassungsrang zu. Insoweit untersagt die Unschuldsvermutung zum einen die Verhängung von Maßnahmen im konkreten Strafverfahren gegen den Beschuldigten, die ohne prozessordnungsgemäßen Schuldnachweis erfolgen und in ihrer Wirkung einer Strafe gleichkommen. Zum anderen gebietet sie den rechtskräftigen Nachweis der Schuld, bevor diese dem Verurteilten im allgemeinen Rechtsverkehr vorgehalten werden darf. ${ }^{9}$

Das Bundesverfassungsgericht bestimmt die Verfassungsmäßigkeit von Medienberichterstattungen über Strafverfahren seit dem Lebach-Urteil im gleichgewichtigen Spannungsfeld von Presse-, Rundfunk- und Filmfreiheit gem. Art. 5 Abs. 1 Satz 2 GG und dem von Art. 2 Abs. 1 GG iVm. Art. 1 Abs. 1 GG gewährleisteten Schutz der Persönlichkeit. ${ }^{10}$ Einerseits bedeutet eine öffentliche Berichterstattung über eine Straftat unter Namensnennung, Abbildung oder Darstellung des Täters stets einen erheblichen Eingriff in dessen Persönlichkeitsrecht. Andererseits ist es Aufgabe der Medien, auch über Straftaten als Bestandteile des Zeitgeschehens zu berichten. ${ }^{11}$ Das gilt umso stärker, je mehr sich die konkrete Straftat hinsichtlich Begehungsart, Besonderheit des Angriffsobjekts oder Schwere der Folgen über die gewöhnliche Kriminalität heraushebt. Insbesondere bei schweren Gewaltverbrechen, gibt es daher neben allgemeiner Neugier und Sensationslust ernstzunehmende Gründe für das Interesse an Information“ über die Tat, die Täter und ihre Motive, ,um sie zu ermitteln und zu bestrafen und um gleichartige Delikte zu verhüten." 12

Wie das Spannungsfeld konkret auszutarieren ist, richtet sich nach dem Bereich, dem die veröffentlichte Information angehört, und der Art der Berichterstattung. Einen absoluten, abwägungsfreien Schutz genießt die Persönlichkeit im Kernbereich höchstpersönlicher privater Lebensgestaltung, namentlich der Intimsphäre, zu der vor allem Ausdrucksformen der Sexualität gehören. Dagegen ist die Veröffentlichung von Informationen aus der bloßen Privatsphäre einer Abwägung unter Beachtung des Verhältnismäßigkeitsgrundsatzes zugänglich, wobei die Prominenz des Beschuldigten zugunsten

$8 \mathrm{Zu}$ weiteren Normtexten SK-Paeffgen, StPO, Bd. X, 4. Aufl. 2012, Art. 6 EMRK Rn. 175 ff.

9 BVerfG NJW 2013, 1058 (1061) unter Bezug auf BVerfGE 19, 342 (347) und 74, 358 (371).

10 BVerfGE 35, 202 (219 ff.); BVerfG NJW 2009, 350 (351 f.); BVerfG ZUM 2010, 243 (245 ff.).

11 BVerfGE 35, 202 (226 ff.); BVerfG ZUM 2010, 243 (245 f.).

12 BVerfGE 35, 202 (231); BVerfG ZUM 2010, 243 (246); auch BVerfG NJW 2009, 350 (351). 
des öffentlichen Informationsinteresses zu berücksichtigen ist. ${ }^{13}$ Das Bundesverfassungsgericht ist der Ansicht, dass bei einer (tages-)aktuellen Berichterstattung über schwere Straftaten das Informationsinteresse der Öffentlichkeit im Allgemeinen dem Persönlichkeitsschutz des Straftäters vorgeht.${ }^{14}$ Die aktuelle Berichterstattung rechtfertigt nicht nur die Namensnennung und ggf. auch die Abbildung des Täters, sondern umgreift auch Informationen über sein persönliches Leben, sofern deren Inhalt in unmittelbarer Beziehung zur Tat steht, über Motive und andere Tatvoraussetzungen Aufschluss gibt und für die Bewertung der Schuld des Täters wesentlich erscheint. ${ }^{15}$

Der Bereich der Sexualität ist insoweit nicht zwangsläufig einer Medienberichterstattung entzogen. Zwar gehört die Freiheit, die eigenen Ausdrucksformen der Sexualität für sich zu behalten, zum unantastbaren Kernbereich privater Lebensgestaltung. Doch unterfällt die Sexualität nicht automatisch diesem Kernbereich. Vielmehr hängt die Zuordnung davon ab, ob ein sexueller Sachverhalt seinem Inhalt nach höchstpersönlichen Charakter hat, inwieweit er in Art und Intensität die Sphäre anderer oder die Belange der Gemeinschaft berührt und ob der Betroffene ihn überhaupt geheim halten will. ${ }^{16}$ Entsprechend sind auch die Umstände der Begehung einer Sexualstraftat einschließlich der Beziehung des Täters zu seinem Opfer, mögen sie auch intime Züge tragen, nicht der absolut geschützten Intimsphäre des Täters zugeordnet. Denn da die Sexualstraftat gewalttätig in die sexuelle Selbstbestimmung und körperliche Unversehrtheit des Opfers eingreift, kann sie nicht als schützenswerter Ausdruck der Persönlichkeitsentfaltung des Täters gem. Art. 2 Abs. 1 GG gewertet werden. ${ }^{17}$

Allerdings ist der Vorrang der Presse-, Rundfunk- und Filmfreiheit bei der Berichterstattung über Straftaten nicht schrankenlos. Das ergibt sich bereits daraus, dass der Persönlichkeitsschutz der Verfahrensbeteiligten in Gerichtsverfahren für das Bundesverfassungsgericht eine über den allgemein in der Rechtsordnung anerkannten Schutzbedarf hinausgehende Bedeutung besitzt. ${ }^{18}$ Deshalb ist eine Namensnennung, Abbildung oder sonstige identifizierende Berichterstattung insbesondere in Fällen der kleinen Kriminalität oder bei jugendlichen Straftätern regelmäßig nicht zulässig. ${ }^{19}$

Auch an die Verdachtsberichterstattung während laufender Ermittlungsverfahren stellt das Bundesverfassungsgericht mit Blick auf die bis zur rechtskräftigen Verurteilung zugunsten des Beschuldigten wirkende Unschuldsvermutung strengere Anforderungen. So wird laut Bundesverfassungsgericht das Persönlichkeitsrecht oftmals bis zu einem erstinstanzlichen Schuldspruch die Freiheit der Berichterstattung überwiegen. Eine individualisierende Berichterstattung kann aber dann gerechtfertigt sein, wenn der Beschuldigte sich nicht mehr mit Relevanz auf sein allgemeines Persönlichkeitsrecht berufen kann. Das wird angenommen bei einer eigenverantwortlichen und gleichfalls

13 BVerfGE 35, 202 (220 f., 232); BVerfG ZUM 2010, 243 (246). Zur Relevanz der auf Neumann-Duesberg zurückgehenden Unterscheidung von absoluten und relativen Personen der Zeitgeschichte Bornkamm NStZ 1983, 102 (104).

14 BVerfGE 35, 202 (231); BVerfG ZUM 2010, 243 (246).

15 BVerfGE 35, 202 (233); BVerfG ZUM 2010, 243 (246).

16 BVerfG ZUM 2010, 243 (246f.).

17 BVerfG ZUM 2010, 243 (247).

18 BVerfG NJW 2001, 1633 (1636); BVerfG NJW 2009, 350 (351).

19 BVerfGE 35, 202 (232); BVerfG ZUM 2010, 243 (246) mit der Gegenausnahme für leichte Verfehlungen bei Besonderheiten in der Person des Täters oder des Tathergangs. 
individualisierenden Stellungnahme des Beschuldigten gegenüber den Tatvorwürfen in den Medien - nicht bei einer Stellungnahme als Zeuge ${ }^{20}$ - oder bei einer besonderen Stellung des Beschuldigten in der Öffentlichkeit durch ein Amt, Prominenz oder sonstige gesellschaftlich hervorgehobene Verantwortung, kraft derer er eine gesteigerte Medienöffentlichkeit hinzunehmen hat. ${ }^{21}$ Stets gebietet die Unschuldsvermutung aber eine zurückhaltende bzw. zumindest ausgewogene Berichterstattung, welche auch die zur Verteidigung des Beschuldigten vorgebrachten Tatsachen und Argumente angemessen berücksichtigt. ${ }^{22}$ Insoweit tritt das Persönlichkeitsrecht des Beschuldigten nur für eine sachbezogene Berichterstattung und seriöse Tatinterpretation zurück, nicht aber für „eine auf Sensationen ausgehende, bewusst einseitige oder verfälschende Darstellung“. 23

Die Unschuldsvermutung verlangt vor allem eine Beachtung der Prangerwirkung, die durch eine identifizierende Berichterstattung ausgelöst werden kann. ${ }^{24}$ Das gilt auch für die Verbreitung von wahren Behauptungen, die ebenfalls Anknüpfungspunkt für soziale Ausgrenzung und Isolierung sein können. ${ }^{25}$ Dabei ist zu berücksichtigen, dass auch eine um Objektivität bemühte Fernsehberichterstattung regelmäßig deutlich stärker in das Persönlichkeitsrecht des Beschuldigten eingreift als eine Wort- und Schriftberichterstattung in Hörfunk und Presse. Insoweit vermag die besondere Schwere von Tatvorwurf und Tatbegehung nicht nur ein gesteigertes Informationsinteresse der Öffentlichkeit zu begründen, sondern auch die Gefahr einer Stigmatisierung des Beschuldigten hervorzurufen, die selbst durch einen Freispruch nicht mehr ausgeräumt werden kann. ${ }^{26}$ Gleichwohl soll sich mit Rücksicht auf die mögliche Prangerwirkung die genaue Grenze einer verantwortungsvollen Medienberichterstattung nur im Einzelfall bestimmen lassen. ${ }^{27}$

Der Bundesgerichtshof für Zivilsachen hat die Grundsätze des Bundesverfassungsgerichts für die Verdachtsberichterstattung weiter eingeengt. Danach kommt eine identifizierende Berichterstattung, insbesondere eine Namensnennung grundsätzlich nur in Fällen schwerer Kriminalität oder bei Straftaten mit besonderer Öffentlichkeitsberührung in Betracht. ${ }^{28}$ Eine zulässige Verdachtsberichterstattung setzt darüber hinaus einen Mindestbestand an Beweistatsachen für den Wahrheitsgehalt der Information voraus, wobei die Medien eine umso höhere Sorgfalt trifft, je schwerer und nachhaltiger die

20 BVerfG NJW 2009, 350 (352).

21 BVerfG ZUM 2010, 243 (246); auch BVerfG NJW 2009, 350 (352); Bornkamm NStZ 1983, 102 (106 f.), der zusätzlich auf den Fall eines gesteigerten Öffentlichkeitsinteresses hinweist, wenn die Presse mit der Veröffentlichung ihrer Kontrollfunktion gegenüber den Ermittlungsbehörden nachkommt.

22 BVerfGE 35, 202 (232); BVerfG NJW 2009, 350 (351).

23 BVerfGE 35, 202 (232).

24 BVerfG NJW 2009, 350 (351).

25 BVerfG ZUM 2010, 243 (245).

26 BVerfG NJW 2009, 350 (352); dazu auch Bornkamm NStZ 1983, 102 (105).

27 BVerfG ZUM 2010, 243 (246).

28 BGH (Z) NJW 2000, 1036 (1038); auch OLG Frankfurt NJW 1980, 597 (598). Wesentlich enger Trüg NJW 2011, 1040 (1042 f.), der nach Verdachtsgraden differenziert und bei einem bloßen Anfangsverdacht jede identifizierende Berichterstattung im Ermittlungsverfahren einschließlich des identifizierenden Hinweises auf die Existenz eines Ermittlungsverfahrens für unzulässig hält. 
Veröffentlichung das Ansehen des Beschuldigten beeinträchtigt. ${ }^{29}$ Regelmäßig wird es zudem erforderlich sein, vor der Veröffentlichung eine Stellungnahme des Betroffenen einzuholen. ${ }^{30}$ Schließlich darf die Berichterstattung nicht präjudizierend bzw. vorverurteilend dahingehend sein, dass sie den unzutreffenden Eindruck erweckt, der Beschuldigten sei der ihm vorgeworfenen Straftat bereits überführt. ${ }^{31}$

\section{Die Rechtsprechung des EGMR}

Die Rechtsprechung des Bundesverfassungsgerichts zum Zusammenhang von Unschuldsvermutung und Medienberichterstattung entspricht im Wesentlichen der Auslegung des Art. 6 Abs. 2 EMRK durch den EGMR. Dabei kommt der Unschuldsvermutung des Art. 6 Abs. 2 EMRK die Funktion einer „Auslegungshilfe“ zur Konkretisierung der inhaltlichen Reichweite des Art. 2 Abs. 1 GG zu. ${ }^{32}$

Nach dem EGMR gilt die Unschuldsvermutung gem. Art. 6 Abs. 2 EMRK als Ausprägung des Rechts auf einen fairen Strafprozess für das gesamte Strafverfahren unabhängig von seinem Ausgang. ${ }^{33}$ Die Anwendbarkeit der Unschuldsvermutung reicht damit vom Vorfeld eines Strafverfahrens über das Ermittlungsverfahren und die Hauptverhandlung bis zum Ende des förmlichen Gerichtsverfahrens. ${ }^{34}$ Die Unschuldsvermutung richtet sich dabei vorrangig nicht nur an die Strafgerichte, sondern ist von allen staatlichen Behörden, insbesondere den Strafverfolgungsbehörden sowie allen Verfahrensbeteiligten $\mathrm{zu}$ beachten. ${ }^{35}$

Die Unschuldsvermutung gewährleistet für den EGMR das Recht, nicht als einer Straftat schuldig bezeichnet oder behandelt zu werden, bevor die Schuld des Betroffenen gerichtlich festgestellt worden ist. ${ }^{36}$ Zwar dürfen die Strafverfolgungsorgane die Öffentlichkeit über laufende Ermittlungs- und Strafverfahren informieren, namentlich auf einen bestehenden Tatverdacht, eine Verhaftung oder das Ablegen eines Geständnisses hinweisen. ${ }^{37}$ Jedoch wird die Unschuldsvermutung verletzt durch Erklärungen und Entscheidungen, die den Eindruck der Schuld des Betroffenen erwecken, die die Öffentlichkeit zu einem Glauben an die Schuld des Betroffenen veranlassen oder die die Tatsachenbeurteilung durch den zuständigen Richter vorwegnehmen. ${ }^{38}$ Die Unschuldsvermutung garantiert m.a.W. die Offenheit des Strafverfahrens bis zum gesetzlichen Schuldnachweis und verbietet, das Strafverfahren als eigene Strafe auszugestalten. ${ }^{39}$

29 BGH (Z) NJW 2000, 1036.

30 BGH (Z) NJW 2000, 1036 (1037).

31 BGH (Z) NJW 2000, 1036 f. Zum Ganzen näher Hohmann NJW 2009, 881 (882 f.).

32 Stellvertretend SK-Paeffgen (Fn. 8), Art. 6 EMRK Rn. 176 mwNw.

33 EGMR NJW 2011, $1789 \mathrm{f}$. - Poncelet/Belgien.

34 Vgl. EGMR HRRS 2009 Nr. 516 Rn. 45 ff. - Borovsky/Slowakei; Barrot ZJS 2010, 701 (702 ff.).

35 EGMR HRRS 2009 Nr. 516 Ziff. 46 - Borovsky/Slowakei; Karpenstein/Mayer-Meyer, EMRK, 2012, Art. 6 Rn. 164; Frowein/Peukert-Peukert, EMRK, 3. Aufl. 2009, Art. 6 Rn. 267; Frowein, FS Huber, 1981, 554; Barrot ZJS 2010, 701.

36 EGMR NJW 2011, 1789 (1790) - Poncelet/Belgien.

37 Frowein/Peukert-Peukert (Fn. 34), Art. 6 Rn. 267; Frowein, FS Huber, 554.

38 EGMR NJW 2011, 1789 (1790) - Poncelet/Belgien; Barrot ZJS 2010, 703.

39 Vgl. zu diesen Aspekten Stuckenberg ZStW 111 (1999), 452 ff. 
Entscheidend für den EGMR ist insoweit die Abgrenzung zwischen zulässiger Verdachtsmitteilung und unzulässiger Vorverurteilung. ${ }^{40}$

Darüber hinaus statuiert Art. 6 Abs. 2 EMRK nach herrschender Ansicht auch eine Schutzpflicht des Staates, vorverurteilenden Berichterstattungen durch die Medien entgegenzutreten. So hält der EGMR den Staat neben der eigenen sorgfältigen Berichterstattung durch seine Strafverfolgungsorgane für verpflichtet, die gesetzlichen Voraussetzungen für eine sachliche und soweit wie möglich objektive Berichterstattung durch die Medien zu schaffen. ${ }^{41}$ Bei gröblich entstellenden Tatsachendarstellungen sollen Justizbehörden sogar zur Abgabe sachlicher Gegendarstellungen aufgerufen sein. ${ }^{42}$

\section{Mittelbare Drittwirkung}

Letzterer Aspekt berührt die Frage, ob und inwieweit der Unschuldsvermutung gem. Art. 6 Abs. 2 EMRK eine Drittwirkung zukommt. Nach herrschender Auffassung entfaltet die Unschuldsvermutung keine unmittelbare Drittwirkung für private Dritte. ${ }^{43}$ Dagegen ist in der Rechtsprechung des Bundesverfassungsgerichts und im überwiegenden Schrifttum anerkannt, dass die Unschuldsvermutung als Menschenrecht und Konventionsgarantie zumindest eine mittelbare Drittwirkung besitzt. ${ }^{44}$

Danach können die Handlungen Dritter wie vor allem der Medien und sonstiger Normalbürger auch nach Maßgabe der Rechte aus Art. 6 Abs. 2 EMRK geprüft werden. ${ }^{45}$ Auch wenn der Gehalt der Unschuldsvermutung dabei nicht absolut gilt, fließt er doch in die aufgezeigte Abwägung zwischen der Presse-, Rundfunk- und Filmfreiheit und dem Persönlichkeitsrecht des Beschuldigten in dem Sinne ein, dass sie das Informationsinteresse der Öffentlichkeit im beschriebenen Maße begrenzt und mäßigt. ${ }^{46}$

Keine Zustimmung verdient insoweit eine Entscheidung des OLG Frankfurt aus dem Jahr 1979. Danach soll die Presse einen Beschuldigten schon im Ermittlungsverfahren als Täter bezeichnen dürfen, wenn

40 Exemplarisch EGMR HRRS 2009 Nr. 516 Ziff. 46 - Borovsky/Slowakei; dazu auch Frowein, FS Huber, 555.

41 EGMR, Urt. v. 28.10.2004 - 48173/99, Ziff. 48 - Y.B. u.a./Türkei; Karpenstein/MayerMeyer (Fn. 35), Art. 6 Rn. 165; SK-Paeffgen (Fn. 8), Art. 6 EMRK Rn. 199; LR-Esser, StPO, 11. Bd. 26. Aufl. 2012, Art. 6 EMRK Rn. 460; Frowein, FS Huber, 556 f.; Barrot ZJS 2010, $703 \mathrm{f}$.

42 So Karpenstein/Mayer-Meyer (Fn. 35), Art. 6 Rn. 165; ebenso im Ergebnis, aber mit anderer Richtung, Janisch NJW aktuell 19/2010, 18.

43 LR-Esser (Fn. 41), Art. 6 EMRK Rn. 456; SK-Paeffgen (Fn. 8), Art. 6 EMRK Rn. 197; Bornkamm NStZ 1983, 102 (104); Stuckenberg ZStW 111 (1999), 459; OLG Frankfurt NJW 1980, 597 (598 f.). A.A. z.B. Marxen GA 1980, 373, der eine unmittelbare Außenwirkung der Unschuldsvermutung auch im Verhältnis zwischen den Medien und dem einzelnen Bürger bejaht; auch wohl Eb. Schmidt (Fn. 1), S. 56.

44 Vgl. nur die oben in B I behandelten Entscheidungen BVerfGE 35, 202 (232); BVerfG NJW 2009, 350 (351); BVerfG ZUM 2010, 243 (246); ferner Karpenstein/Mayer-Meyer (Fn. 35), Art. 6 Rn. 166; LR-Esser (Fn. 41), Art. 6 EMRK Rn. 458; SK-Paeffgen (Fn. 8), Art. 6 EMRK Rn. 198; Bornkamm NStZ 1983, 102 (104). Zweifelnd aber BGH (Z) NJW 2000, 1036 (1037).

45 Zutreffend OLG Frankfurt NJW 1980, 597 (599) m. Bspr. Grave NJW 1981, 209.

46 Vgl. oben B I, insbesondere BVerfGE 35, 202 (232); BVerfG NJW 2009, 350 (351); BVerfG ZUM 2010, 243 (246); weicher OLG Frankfurt NJW 1980, 597 (599). 
(1) ihre Recherchen zu einem so gravierenden Verdacht einer schweren, für das öffentliche Interesse bedeutsamen Straftat geführt haben, dass sie von der Richtigkeit der sorgfältig überprüften Beschuldigungen überzeugt ist,

(2) die Umstände des Falles zu einer deutlichen Anprangerung des Betroffenen als Täter drängen und

(3) aus dem Text der Veröffentlichung hervorgeht, dass eine strafrechtliche Verurteilung nicht erfolgt ist. ${ }^{47}$

Diese Entscheidung trägt den Grundsätzen der Unschuldsvermutung, wie sie sowohl das Bundesverfassungsgericht als auch der EGMR herausgearbeitet haben, nicht hinreichend Rechnung. Die Unschuldsvermutung gebietet vor dem gesetzlichen Nachweis der Schuld die strikte Trennung von zulässiger Tatverdachtsmitteilung und unzulässiger, weil vorverurteilender Täterdarstellung. Alle drei Voraussetzungen, von denen das Gericht die Zulässigkeit der Täterdarstellung abhängig macht, sind nicht tragfähig. Erstens kann die besondere Schwere des Tatvorwurfs eine Täterdarstellung bereits im Ermittlungsverfahren nicht rechtfertigen, weil nach dem Bundesverfassungsgericht gerade durch sie zugleich die Gefahr einer besonders schweren Stigmatisierung des Beschuldigten bewirkt werden kann. ${ }^{48}$ Zweitens findet die zulässige Prangerwirkung ihre Grenze in der identifizierenden Berichterstattung über den Tatverdächtigen. Und drittens ist der bloße Hinweis auf das Fehlen einer strafrechtlichen Verurteilung in der Veröffentlichung nicht geeignet, juristischen Laien den von der Unschuldsvermutung gebotenen Unterschied zwischen Tatverdacht und Tatschuld zu vermitteln. ${ }^{49}$

\section{Gezielte Öffentlichkeit: Drei Beispielsfälle}

Auf Basis der vorstehend rekonstruierten Rechtsprechung des Bundesverfassungsgerichts und des EGMR zur Unschuldsvermutung können drei Beispiele einer, ,gezielten Öffentlichkeit" durch Strafverfolgungsorgane, Medien und Private einer Kritik zugeführt werden. Untersucht werden die Fälle Benaissa, Kachelmann und Emden.

\section{1. Öffentliche Inszenierung der Verhaftung. Der Fall Benaissa}

Dem Fall Benaissa lag folgender Sachverhalt zugrunde: Am Abend des 11. April 2009 wurde die ehemalige No-Angels-Sängerin Nadja Benaissa kurz vor einem geplanten Solokonzert in einer Diskothek in Frankfurt am Main wegen des Vorwurfs der gefährlichen Körperverletzung verhaftet und in Untersuchungshaft genommen. Wie die Staatsanwaltschaft Darmstadt wenig später in einer Pressemitteilung erklärte, bestand der dringende Tatverdacht, dass die Verhaftete, eine 26jährige Sängerin, in den Jahren 2000 und 2004 ungeschützten Sex mit mehreren Personen hatte, ohne diese auf ihre HIVInfektion hingewiesen zu haben. Zumindest einer der Sexualpartner sei mutmaßlich von ihr mit HIV angesteckt worden. Als Haftgrund nannte die Staatsanwaltschaft Wiederholungsgefahr. Obwohl die Pressemitteilung keinen Namen angibt, war klar, wer ge-

47 OLG Frankfurt NJW 1980, 597 (598) m. abl. Bspr. Grave NJW 1981, 209.

48 Vgl. oben II. 1. mit Fn. 26; zu dieser Kritik auch Grave NJW 1981, 210.

49 Weitere Kritik bei Grave NJW 1981, 209 ff. 
meint war, nachdem die Bild-Zeitung am Morgen der Pressemitteilung über die Verhaftung von Frau Benaissa berichtet hatte. Die Polizei hatte die öffentliche Verhaftung damit begründet, dass Frau Benaissa nicht anders erreichbar gewesen sei. Bereits zehn Tage später wurde die Beschuldigte aus der Untersuchungshaft gegen die Erfüllung von Auflagen entlassen. Eine am 14. April 2009 beim Landgericht Berlin erwirkte einstweilige Verfügung gegen die Bild-Zeitung auf Unterlassung der Berichterstattung zog die Beschuldigte Ende Mai 2009 aus vorgeblich strategischen Gründen zurück. Im August 2010 wurde Frau Benaissa wegen vollendeter und versuchter gefährlicher Körperverletzung zu zwei Jahren Freiheitsstrafe auf Bewährung und 300 Stunden gemeinnützige Arbeit verurteilt. ${ }^{50}$

Das Vorgehen der Strafverfolgungsbehörden im Fall Benaissa ist mit Blick auf die Unschuldsvermutung in Politik, Justiz und Rechtswissenschaft heftig kritisiert worden. Gerügt wurde vor allem, dass die Sängerin in der Öffentlichkeit verhaftet worden sei und dass die Staatsanwaltschaft die Medien über ihre HIV-Infektion informiert habe. ${ }^{51}$ Was letzteren Vorwurf betrifft, so erscheint die Sache nicht eindeutig. ${ }^{52}$ Wendet man die Grundsätze von Bundesverfassungsgericht und EGMR an, so sprechen für die Zulässigkeit der staatsanwaltschaftlichen Pressemitteilung die Schwere des Tatvorwurfs und der Tatfolgen; die Prominenz der Beschuldigten; der Umstand, dass die Beschuldigte zum Zeitpunkt der Haupttat Erwachsene war ${ }^{53}$; die grundsätzliche Zulässigkeit der Berichterstattung auch über Sexualität; und der konkrete Tatbezug der HIV-Infektion der Beschuldigten. ${ }^{54}$ Gegen die Zulässigkeit der Presseerklärung in dieser Form streiten andererseits die mit der Schwere des Tatvorwurfs verbundene Stigmatisierungsund Prangerwirkung; der frühe Zeitpunkt der Mitteilung; der Umstand, dass die Beschuldigte bei einem Teil der vorgeworfenen Taten noch Heranwachsende war; das Nichtvorliegen eines Sexualdelikts im engeren Sinn; und die Zugehörigkeit des Tatumstands der HIV-Infektion zum Intimbereich. Wägt man diese Umstände im Lichte der betroffenen Grundrechte gegeneinander ab und berücksichtigt, dass die Staatsanwaltschaft hinsichtlich des $\mathrm{Ob}$ und des Zeitpunkts der Veröffentlichung einen Spielraum hat $^{55}$, so halte ich die wahrheitsgemäße, nicht reißerische Pressemitteilung der Staats-

50 Vgl. zum Sachverhalt die Berichte bei Stern.de v. 16.4.2009; SpiegelOnline v. 21.4.2009; Frankfurter Rundschau v. 29.4.2009; MEEDIA v. 27.5.2009; Süddeutsche.de v. 27.10.2010; ferner die Presseerklärung der StA Darmstadt vom 12.2.2010 zur Anklageerhebung im Fall Nadja B.

51 Siehe z.B. Stern.de v. 16.4.2009; SpiegelOnline v. 21.4.2009; Frankfurter Rundschau v. 29.4.2009; Ladeur AfP 2009, 446 (449); Prantl AnwBl. 6/2009, 421; Zabel GA 2011, 347 (354-359).

52 Es erscheint entgegen der anderslautenden Bekundung nicht ausgeschlossen, dass diese Uneindeutigkeit auch beim Zurückziehen der erstrittenen einstweiligen Verfügung eine Rolle gespielt haben könnte.

53 Vgl. Süddeutsche.de v. 27.10.2010.

54 Zur Relevanz der beiden letzten Aspekte oben II. 1.

55 Vgl. Einführung RiStBV iVm. Nr. 23 RiStBV zur Zusammenarbeit mit Presse und Rundfunk; dazu Dalbkermeyer, Der Schutz des Beschuldigten usw., 1994, S. 25, 32 ff. 
anwaltschaft in dieser Form und zu diesem frühen Zeitpunkt nicht für gänzlich unvertretbar. 56

Anders ist die öffentliche Verhaftung von Frau Benaissa kurz vor ihrem Konzert in der Diskothek zu bewerten. Wie der präventiv-polizeiliche ${ }^{57}$ und insoweit problematische Haftgrund der Wiederholungsgefahr gem. § 112 a StPO vorgibt, sahen die Ermittlungsbehörden nicht nur bestimmte Tatsachen als gegeben an, die die Gefahr begründeten, dass die Beschuldigte weitere erhebliche Straftaten gleicher Art begehen würde. Sie bejahten auch die Erforderlichkeit der Haft zur Abwendung der drohenden Gefahr. Warum die konkrete Verhaftung der Sängerin allerdings in dieser für sie maximalen (Unterhaltungs-) Öffentlichkeit ${ }^{58}$ erfolgen musste, ist von den Zwecken des $§ 112$ a StPO nicht mehr gedeckt. Soweit die Polizei angibt, die Beschuldigte sei anders nicht erreichbar gewesen ${ }^{59}$, erscheint das vorgeschoben. Der beziehungsreichen Wahl des Verhaftungsortes (Konzert der Sängerin) dürfte vielmehr der generalpräventive Zweck zugrunde gelegen haben, der (Unterhaltungs-)Öffentlichkeit die Gefährlichkeit von Aids vor Augen zu führen. ${ }^{60}$ Damit verfolgte die Verhaftung aber einen allgemein-repressiven Strafzweck, der von den Sicherungszwecken der Untersuchungshaft im konkreten Verfahren nicht getragen war. ${ }^{61}$ Insoweit wurde die öffentliche Verhaftung allein zu verfahrensfremden Prangerzwecken missbraucht und die Beschuldigte entgegen Nr. 23 Abs. 1 RiStBV ,gezielt“ bloßgestellt. ${ }^{62}$ Verhaftungen dürfen aber nicht so vorgenommen werden, dass wegen der mit ihnen verbundenen Prangerwirkung der Eindruck eines überführten Straftäters entsteht. Immerhin ist das Ermittlungsverfahren grundsätzlich nicht öffentlich. ${ }^{63}$ Mit Recht gelten deshalb auch Verhaftungen von Prominenten vor laufenden Fernsehkameras, die durch gezielte Indiskretionen von Strafverfolgungsbehörden ermöglicht werden, regelmäßig als Verstoß gegen die Unschuldsvermutung. ${ }^{64}$ Soweit die öffentliche Verhaftung von Frau Benaissa ebenfalls strafähnliche Wirkung hatte, wurde die Beschuldigte bereits im Ermittlungsverfahren als Schuldige instrumentalisiert und folglich ihre Unschuldsvermutung verletzt. ${ }^{65}$

56 A.A. Prantl AnwB1. 6/2009, 421. Vgl. zu den Zulässigkeitsanforderungen an staatsanwaltliche Pressemitteilungen Raschke ZJS 2011, 44 ff.; ferner die Stellungnahme von Janisch NJW aktuell 19/2010, 16.

57 Meyer-Goßner, StPO, 55. Aufl. 2012, § 112 a Rn. 1.

58 Begriff von Zabel GA 2011, 355.

59 Vgl. Frankfurter Rundschau v. 29.4.2009.

60 Prantl AnwB1. 6/2009, 421.

61 Vgl. zu diesen Zwecken statt aller Meyer-Goßner (Fn. 57), Vor § 112 Rn. 4.

62 Gem. Nr. 23 Abs. 1 S. 4 RiStBV ist eine unnötige Bloßstellung des Beschuldigten zu vermeiden; dazu Dalbkermeyer (Fn. 55), S. $33 \mathrm{ff}$.

63 Ostendorf/Frahm/Doege NStZ 2012, 529 (530).

64 Zutreffend Boehme-Neßler ZRP 2009, 228 (229).

65 Im Ergebnis ebenso Prantl AnwBl. 6/2009, 421; Zabel GA 2011, 356 ff. 


\section{Grenzenlose Medienberichterstattung? Der Fall Kachelmann}

Fraglich ist, ob die Medienberichterstattung im Fall Kachelmann ein weiteres Anwendungsbeispiel einer unzulässigen ,gezielten Öffentlichkeit“ darstellt. ${ }^{66}$ Von der komplexen und umfänglichen Medienberichterstattung in diesem Fall sei folgender Sachverhalt ausgewählt, der Gegenstand einer Zivilklage gewesen ist:

Der Fernsehmoderator und Journalist Jörg Kachelmann wurde im März 2010 wegen des Vorwurfs der Vergewaltigung einer damaligen Freundin in einem besonders schweren Fall in Tateinheit mit gefährlicher Körperverletzung verhaftet und in Untersuchungshaft genommen. Bereits früh wurden aus ungeklärter Quelle Passagen aus der Vernehmung des Beschuldigten vor dem Ermittlungsrichter am 24.3.2010 in den Medien bekannt. Am 13.7.2010, also nach Anklageerhebung und vor Eröffnung des Hauptverfahrens, berichtete das Internetportal www.bild.de unter der Überschrift „Der K...Krimi. Neue Indizien aus der Tatnacht?“ intime Details aus der sexuellen Beziehung zwischen dem Beschuldigten und dem vermeintlichen Tatopfer, insbesondere über einvernehmliche sado-masochistische Praktiken. Die Informationen stammten ebenfalls aus der Einlassung des Beschuldigten vor dem Ermittlungsrichter. Das Protokoll dieser Vernehmung wurde in der öffentlichen Hauptverhandlung am 13.9.2010 zu Beweiszwecken verlesen. Bereits Ende Juli 2010 war der Beschuldigte aus der Untersuchungshaft entlassen worden. Am 31. Mai 2011 hat ihn das Landgericht Mannheim aus Mangel an Beweisen freigesprochen. ${ }^{67}$

Das Verhältnis von Medien und Strafprozess ist im Fall Kachelmann wegen der dort zu Tage getretenen scheinbar grenzenlosen Berichterstattung bereits von verschiedener Seite einer kritischen Betrachtung unterzogen worden. ${ }^{68}$ Hier soll allein geprüft werden, ob die Berichterstattung im Internetportal www.bild.de vom 13.6.2010 die Unschuldsvermutung des Betroffenen verletzt hat. Das $O L G$ Köln bejaht - die mittelbare Drittwirkung der Unschuldsvermutung für die Medien voraussetzend ${ }^{69}$ - diese Frage unter eingehender Würdigung der dargestellten Rechtsprechung des Bundesverfassungsgerichts und bestätigt den vom LG erkannten Anspruch des Beschuldigten auf Unterlassung der Veröffentlichung und Verbreitung. ${ }^{70}$ Zunächst erkennt das Gericht zutreffend, dass eine Abwägung zwischen den widerstreitenden Grundrechten eröffnet ist, weil der Tatvorwurf ein Sexualdelikt betrifft und die veröffentlichten Informationen deshalb nicht zum absolut geschützten Kernbereich des Persönlichkeitsrechts gehören. ${ }^{71}$ Bei der Abwägung gewichtet das OLG Köln das Persönlichkeitsrecht des Beschuldigten mit Fug höher als das Berichterstattungsinteresse des Internetportals. Zentral ist hierbei die Feststellung, dass die fragliche Berichterstattung keinen konkreten Bezug zu den Tatvorwürfen aufweist, sondern in reißerischer Weise vorrangig der Darstellung angebli-

66 Reiches Fallmaterial zu Vorverurteilungen in der Presse zwischen 1985 und 1997 bei Soehring, Vorverurteilung durch die Presse, 1999, S. 157 ff.

67 Vgl. zum Sachverhalt den Tatbestand in BGH Urt. v. 19.3.2013 - Az. VI ZR 93/12, Rn. 2 ff.

68 Siehe etwa Friedrichsen/Gerhardt (Fn. 1), 10. Teil Kap. 2, Rn. 36 a ff.; Jung JZ 2012, 303; Rückert StV 2012, 378.

69 Dazu oben II. 3.

70 OLG Köln ZUM-RD 2012, 206 (209 ff. unter maßgeblichem Bezug auf BVerfG ZUM 2010, 243 und auch BVerfG NJW 2009, 350) m. zust. Bspr. Gostomzyk AfP 2012, 122.

71 OLG Köln ZUM-RD 2012, 206 (209). 
cher sexueller Vorlieben des Beschuldigten dient. Insoweit fehlt nicht nur ein legitimes Informationsinteresse. Vielmehr gebietet die Unschuldsvermutung eine besondere $\mathrm{Zu}$ rückhaltung und Ausgewogenheit der Berichterstattung, welche die streitige Berichterstattung gerade nicht wahrt. ${ }^{72}$ Hinzu kommt, dass die Prangerwirkung durch eine mit dem Tatvorwurf nicht zusammenhängende Berichterstattung im sexuellen Bereich für den Beschuldigten im besonderen Maße stigmatisierend wirkt und damit strafähnlichen Charakter hat. ${ }^{73}$

Die Verletzung der Unschuldsvermutung ist nach Auffassung des OLG Köln nicht dadurch mit der Folge der Zulässigkeit der Berichterstattung entfallen, dass die vermeintlichen Sexualpraktiken des Beschuldigten später in anderen Medien publik geworden sind. Denn den Störer kann das - möglicherweise - rechtswidrige Verhalten Dritter nicht entlasten. ${ }^{74}$ Der Beschuldigte hat sich auch nicht in eigenverantwortlicher Weise selbst des Schutzes seines Persönlichkeitsrechts begeben. Insbesondere ist er nicht mit den fraglichen Details aus seinem Intimleben an die Öffentlichkeit getreten. ${ }^{75}$ Keine andere Beurteilung rechtfertigt nach Ansicht des OLG Köln vor allem die spätere Verlesung des Protokolls seiner richterlichen Vernehmung in der öffentlichen Hauptverhandlung. Denn die Medienberichterstattung über diese Hauptverhandlung müsse die gleiche Zurückhaltung und Ausgewogenheit zeigen wie die Verdachtsberichterstattung, so dass sie über die nicht tatbezogenen sexuellen Vorlieben des Beschuldigten nicht berichten durfte. Das Gericht begründet die Einschränkung der mittelbaren Medienöffentlichkeit der Hauptverhandlung mit einer kreativen Analogie: „Die in diesem Zusammenhang von dem BVerfG angeführten Erwägungen dazu, inwieweit Persönlichkeitsrechte durch eine Fernseh- oder Rundfunkübertragung des Prozesses beeinträchtigt werden, müssen gleichermaßen auf die Frage erstreckt werden, inwieweit Persönlichkeitsrechte der Beteiligten auch bei sonstigen Formen der Berichterstattung über ein Gerichtsverfahren zu achten sind." ${ }^{76}$ M.a.W.: Saalöffentlichkeit und Medienöffentlichkeit sind zwei verschiedene Dinge. Schließlich trägt auch der Aspekt der Kritik an der Lebensführung einer prominenten Person nicht die beanstandete Berichterstattung. Denn selbst für prominente Beschuldigte gilt der Schutz der Intim- und Sexualsphäre. ${ }^{77}$

Dass die streitige Berichterstattung im Fall Kachelmann im Zeitpunkt ihrer Veröffentlichung rechtswidrig gewesen ist, hat der 6. Zivilsenat des $B G H$ in seinem Revisionsurteil vom März 2013 bestätigt. ${ }^{78}$ Der BGH billigt ausdrücklich die vom OLG Köln in Ansatz gebrachten rechtlichen Grundsätze, die Zuordnung der streitigen Berichterstattung zur Privatsphäre des Beschuldigten und den angenommenen Vorrang des Persönlichkeitsrechts und der Unschuldsvermutung. ${ }^{79}$ Von besonderem Gewicht zugunsten des Beschuldigten wertet der BGH vor allem die belastenden Umstände, dass die fraglichen Äußerungen aus der Einlassung des Beschuldigten bei der haftrichterlichen Ver-

72 OLG Köln ZUM-RD 2012, 206 (210).

73 OLG Köln ZUM-RD 2012, 206 (211).

74 OLG Köln ZUM-RD 2012, 206 (211).

75 OLG Köln ZUM-RD 2012, 206 (211 f.).

76 OLG Köln ZUM-RD 2012, 206 (212) unter Rekurs auf BVerfG NJW 2001, 1633 (1636).

77 OLG Köln ZUM-RD 2012, 206 (213).

78 BGH NJW 2013, 1681 (1682 Rn 20).

79 BGH NJW 2013, 1681 ff. (Rn 15 ff., 21 ff., 27 ff.). 
nehmung stammten, die nicht öffentlich und daher auch für Medien unzugänglich ist, und dass die streitige Berichterstattung noch vor Beginn der Hauptverhandlung erfolgte. ${ }^{80}$ Mit Recht stellt der BGH zudem klar, dass wahre Tatsachenbehauptungen die Beachtung der Unschuldsvermutung im Rahmen der Abwägung nicht ausschließen. ${ }^{81}$ Insoweit kann im Fall Kachelmann die streitgegenständliche Berichterstattung vom Juli 2010 zumindest im Zeitpunkt ihrer Veröffentlichung als Beispiel einer die Unschuldsvermutung verletzenden ,gezielten Öffentlichkeit“" angesehen werden.

Allerdings erachtet der BGH den Unterlassungsanspruch von Kachelmann anders als das OLG Köln im Ergebnis für nicht begründet. Denn nach Verlesung des Protokolls über die haftrichterliche Vernehmung in der öffentlichen Hauptverhandlung sei die für den Unterlassungsanspruch erforderliche Wiederholungsgefahr entfallen. ${ }^{82}$ Insofern müsse berücksichtigt werden, dass die Berichterstattung über diese Hauptverhandlung, welche die beanstandeten Details einschloss, als tagesaktuelle Berichterstattung auch mit Blick auf die Unschuldsvermutung zulässig gewesen sei. Insbesondere weise die spätere Berichterstattung nun den notwendigen konkreten Tatbezug auf, weil „die Einlassung von zentraler Bedeutung für die Berichterstattung und für die öffentliche Meinungsbildung hinsichtlich eines möglichen Geschehensablaufs in der Tatnacht und die Beurteilung der Glaubwürdigkeit der Beteiligten" war. Deshalb bestehe keine Wiederholungsgefahr mehr. ${ }^{83}$ Das Urteil des BGH hinterlässt den fahlen Beigeschmack, dass zunächst nicht konkret tatbezogene intime Details letztlich doch sanktionslos verbreitet werden dürfen. Damit könnte dem Sujet des Schlüpfrigen wider die Unschuldsvermutung ein $\mathrm{zu}$ weites Tor geöffnet werden.

\section{Aufruf zur Lynchjustiz: Der Fall Emden}

Die massivste Verletzung der Unschuldsvermutung markiert gewiss der Aufruf zur Lynchjustiz im Emdener Fall. Dabei ging es um folgenden Sachverhalt: Am 24.3.2012 entdeckte ein Wachmann in einem Parkhaus in Emden die Leiche eines elfjährigen Mädchens. Die Polizei bildete eine 40köpfige Sonderkommission „Parkhaus“, verbreitete am Folgetag eine Pressemitteilung und veröffentlichte Videoaufnahmen aus dem Parkhaus. Für Hinweise zur Ergreifung des Täters setzte die Stadt Emden eine Belohnung in Höhe von $10.000 €$ aus. Nach einem Hinweis aus der Bevölkerung verhafteten uniformierte Polizeibeamte am 27.03. mit Handschellen einen 17jährigen Berufsschüler aus Emden in der Wohnung seines Vaters. Der Hinweis stammte von einem 15jährigen Schüler, der den Tatverdächtigen auf dem Überwachungsvideo erkannt haben wollte. Der am selben Abend vernommene Tatverdächtige legte kein Geständnis ab. Nachdem über Facebook Meldungen über die Festnahme verbreitet worden waren, rief ein 18jähriger Ostfriese noch am gleichen Abend via Facebook zur Erstürmung des Emdener Polizeikommissariats und zur Lynchjustiz an dem mutmaßlichen Täter auf. Dem Aufruf folgten etwa 50 Personen, die bis in die Nachstunden lautstark die Herausgabe des Tatverdächtigen unter Parolen wie „Hängt ihn auf, steinigt ihn!“‘ forderten. Bereits am

80 BGH NJW 2013, 1681 (1682 Rn 27 f.).

81 BGH NJW 2013, 1681 (1682 f. Rn 29).

82 BGH NJW 2013, 1681 (1683 Rn 30 ff.).

83 BGH NJW 2013, 1681 (1683 Rn 33). 
30.03. ließ die Polizei den Berufsschüler frei, weil er als Täter ausgeschlossen werden konnte. Der Berufsschüler verließ zunächst seine Heimatstadt. Am 31.03. nahm die Polizei den wahren Täter fest. Das Jugendschöffengericht Emden verwarnte den 18jährigen Ostfriesen und ordnete wegen öffentlicher Aufforderung zu Straftaten zwei Wochen Jugendarrest an. ${ }^{84}$

Die Unschuldsvermutung kann im Emder Fall zumindest in zweifacher Hinsicht als „gezielte Öffentlichkeit“" verletzt worden sein. ${ }^{85}$ Zum einen ist der Vorwurf erhoben worden, dass die Polizei bei der Verhaftung des Berufsschülers nicht mit der gebotenen Diskretion vorgegangen sei. Durch die Verhaftung mit Uniform und Handschellen habe sie in einem kleinstädtischen Umfeld eine unnötige Prangerwirkung erzeugt und damit die Unschuldsvermutung des Berufsschülers unnötig verletzt. ${ }^{86}$ An diesem Vorwurf ist zutreffend, dass bei Minderjährigen wie dem Tatverdächtigen eine besondere Vorsicht und Rücksichtnahme auf die Persönlichkeitsrechte geboten ist. ${ }^{87}$ Auf der anderen Seite ist nicht unzweifelhaft, ob im konkreten Fall schon die schiere Verhaftung eines Minderjährigen in Uniform und Handschellen als unzulässige „öffentliche Bloßstellung“ angesehen werden kann. Offensichtlich waren Medien bei der Verhaftung nicht zugegen. Außerdem zeigt die rasche und intensive Nutzung von Facebook zur Informationsverbreitung im Emdener Fall, dass vermutlich in dem kleinstädtischen Umfeld von Emden vor dem Hintergrund eines unaufgeklärten Mordes an einem Kind Verhaftung und Person des Tatverdächtigen ohnehin rasch bekannt geworden wären. ${ }^{88}$

Evident die Unschuldsvermutung verletzt hat demgegenüber der Aufruf zur Lynchjustiz durch den 18jährigen Ostfriesen. Lynchjustiz hebelt als „kurzer Prozess“ den gesetzlichen Nachweis der Schuld sprichwörtlich aus. Sie stellt damit die denkbar massivste Missachtung der Unschuldsvermutung des Beschuldigten dar. Ähnliches gilt für den Aufruf zur Lynchjustiz als Vorbereitungshandlung, der wie die Lynchjustiz strafbar ist. ${ }^{89}$ Dass der konkrete Aufruf zur Lynchjustiz dem Staat wohl nicht zugerechnet werden kann, da er durch einen Privaten über ein soziales Netzwerk erfolgte ${ }^{90}$, ändert nichts an der Geltung und Missachtung der Unschuldsvermutung im Emdener Fall. Über die mittelbare Drittwirkung ist die Unschuldsvermutung auch im Verhältnis von Privaten zueinander anwendbar. ${ }^{91}$ Gerade der Emdener Fall beweist, wie notwendig es ist, mit der Unschuldsvermutung auch ,im Verhältnis der Staatsbürger untereinander für eine Atmosphäre der Anständigkeit“ zu sorgen ${ }^{92}$ und ,gezielte Öffentlichkeit“ zu unterbinden.

84 Vgl. den Sachverhaltsbericht bei Ostendorf/Frahm/Doege NStZ 2012, 529 f.; ferner Bild.de v. 31.3.2012; Süddeutsche.de v. 1.4.2012 (Großmann/Serrao); SpiegelOnline v. 2.4.2012.

85 Die Prüfung der anschließenden Berichterstattung klammere ich aus. Ostendorf/Frahm/Doege NStZ 2012, 529 (530) sind der Ansicht, dass auch insoweit teilweise gegen die Unschuldsvermutung verstoßen wurde; vgl. auch die Kritik von Heger bei Bild.de v. 31.3.2012.

86 Vgl. SpiegelOnline v. 2.4.2012; im Ergebnis ebenso Ostendorf/Frahm/Doege NStZ 2012, 529 (530).

87 Vgl. oben II. 1. mit Fn. 19.

88 Wie hier Gundlach bei SpiegelOnline v. 2.4.2012.

89 Ostendorf/Frahm/Doege NStZ 2012, 529 (531 ff.) prüfen Strafbarkeiten nach § 111; §§ 212, 26, 30 Abs. 1 S. 1 Alt. 1 ; $\S \S 212,30$ Abs. 2 Var. 3 und $\S 126$ StGB.

90 Ebenso Gundlach bei SpiegelOnline v. 2.4.2012.

91 Dazu oben II. 3.

92 Eb. Schmidt (Fn. 1), S. 56. 


\section{Zusammenfassung}

„Gezielte Öffentlichkeit“ bildet ein offenbar zunehmendes Begleitphänomen moderner Strafverfahren. Sie bezeichnet den Sachverhalt, dass Strafverfolgungsorgane, Medien oder Private sich während laufender strafrechtlicher Ermittlungsverfahren gezielt an die Öffentlichkeit wenden, um die Prangerwirkung des Strafverfahrens zu verfahrensfremden Zwecken zu instrumentalisieren. Gezielte Öffentlichkeit widerstreitet der Unschuldsvermutung und ist daher unzulässig. Der Beitrag analysiert anhand dreier aktueller und prominenter Strafverfahren verschiedene Erscheinungsformen der gezielten Öffentlichkeit: die öffentliche Inszenierung der Verhaftung (Fall Benaissa), eine grenzenlose Medienberichterstattung (Fall Kachelmann) und den Aufruf zur Lynchjustiz (Fall Emden). Die Beispiele unterstreichen, dass die gezielte Öffentlichkeit verschärfter Aufmerksamkeit bedarf, um eine Aushöhlung der Unschuldsvermutung im Zeitalter des Medienstrafverfahrens zu verhindern. 